\title{
Hipovitaminosis D: una visión desde la clínica y la biologia molecular
}

\author{
Daniel Gallego-González* \\ Susana Mejía-Mesa** \\ Lina María Martínez-Sánchez ${ }^{* * *}$ \\ Manuela Rendón-Diez ${ }^{* * * *}$
}

*Estudiante de X semestre de medicina. Grupo de Investigación de Ginecología. Universidad Pontificia Bolivariana. Facultad de Medicina. Medellín. Antioquia. Colombia.

**Estudiante de VIII semestre de medicina. Grupo de Investigación de Ginecología. Universidad Pontificia Bolivariana. Facultad de Medicina. Medellín. Antioquia. Colombia.

***Bacterióloga. Especialista en Hematología y Manejo del Banco de Sangre. Magíster. Docente titular. Grupo de Investigación de Ginecología. Universidad Pontificia Bolivariana. Facultad de Medicina. Medellín. Antioquia. Colombia.

****Estudiante de IX semestre de medicina. Grupo de Investigación de Ginecología. Universidad Pontificia Bolivariana. Facultad de Medicina. Medellín. Antioquia. Colombia.

Correspondencia: Daniel Gallego González. Universidad Pontificia Bolivariana, sede Robledo, Medellín, Colombia. Teléfono: +57(4) 4936300. Ext: 854. Correo electrónico: daniel.gallegogo@upb.edu.co.

\section{RESUMEN}

La vitamina $\mathrm{D}$ es una hormona que participa en gran cantidad de procesos fisiológicos y bioquímicos dentro del organismo humano, tales como la absorción de calcio y fosfato a nivel intestinal, regulación de la calcemia y mineralización ósea; además, tiene efectos sobre el ciclo celular, proliferación, diferenciación, señalización, apoptosis y producción de varias moléculas en diferentes células y tejidos, lo que explica la relación de sus bajos niveles con el desarrollo de múltiples patologías. En los últimos años, se ha reportado una elevada prevalencia de hipovitaminosis D a nivel mundial, que oscila entre el 50 y 92\% según la población estudiada, producto de la deficiente exposición a la luz solar, escasa ingesta dietética, poco uso de suplementos vitamínicos, entre otros factores asociados. A su vez, se ha encontrado que la deficiencia de vitamina $\mathrm{D}$ es causada por algunos polimorfismos genéticos que afectan su metabolismo y conllevan al desarrollo de varias enfermedades. Debido a la importancia clínica de la vitamina D, se realizó esta revisión de la literatura por medio de la búsqueda de artículos originales, revisiones sistemáticas y narrativas en las bases de datos Pubmed y ScienceDirect y en el buscador Google Scholar. Se seleccionaron en total 71 artículos, a partir de los cuales se busca describir en el presente texto algunos de los aspectos relacionados con la fisiología, epidemiología, clínica y etiología de la hipovitaminosis D. MÉD.UIS. 2017;30(1):45-56.

Palabras clave: Deficiencia de vitamina D. Factores de riesgo. Endocrinología.

\section{Vitamin D deficiency: a view from clinical features and molecular biology}

\begin{abstract}
Vitamin $D$ is a hormone involved in many physiological and biochemical processes within the human, such as the absorption of calcium and phosphate in the intestine, regulation of blood calcium and bone mineralization; it also has effects on the cell cycle, proliferation, differentiation, signaling, apoptosis and production of various molecules in different cells and tissues, which explains the relationship between low levels of vitamin $D$ and the development of many diseases. In recent years, there has been reported a high prevalence of vitamin D deficiency worldwide, ranging between 50 and 92\% depending on the population studied, the result of poor exposure to sunlight, poor dietary intake, little use of vitamin supplements, among other associated factors. Hence, it has been found that vitamin D deficiency is caused by some genetic polymorphisms that affect their metabolism and lead to the development of various diseases. Because of the clinical importance of vitamin D, this review of the literature was conducted by the search for original articles, systematic reviews and narrative articles in PubMed, ScienceDirect and Google Scholar reviews. They were selected in total 71 articles, from which we seek to describe here some of the aspects of physiology, epidemiology, clinical and etiology of vitamin D deficiency. MÉD.UIS. 2017;30(1):45-56.
\end{abstract}

Keywords: Vitamin D deficiency. Risk factors. Endocrinology.

Artículo recibido el 05 de Marzo de 2016 y aceptado para publicación el 27 de Junio de 2016 
¿Cómo citar este artículo?: Gallego-González D, Mejía-Mesa S, Martínez-Sánchez LM, Rendón-Diez M. Hipovitaminosis D: una visión desde la clínica y la biología molecular. MÉD.UIS. 2017;30(1):45-56.

\section{INTRODUCCIÓN}

La vitamina $D$ es una hormona esteroidea liposoluble que participa en gran cantidad de procesos metabólicos ampliamente descritos en la literatura, como lo son la absorción de calcio y fosfato a nivel intestinal,laregulacióndelacalcemia,lamineralización ósea, entre otros ${ }^{1,2}$. Estudios recientes han revelado el importante papel que desempeña esta vitamina en otros sistemas, ya que se ha evidenciado que está involucrada en la función del páncreas y músculo liso; actúa como protectora e inmunomoduladora en patologías como la hipertensión arterial, cáncer, osteoartritis, esclerosis múltiple, diabetes mellitus y enfermedades infecciosas; adicionalmente, participa en procesos autocrinos, paracrinos, endocrinos, de proliferación, diferenciación y vías de señalización celular, asociadas al calcio y especies reactivas de oxígeno $^{1-3}$. Así mismo, se ha reportado que la vitamina D cumple varias acciones en el sistema inmune, pues está implicada en el aumento de la producción de linfocitos y en la disminución de la producción de citoquinas proinflamatorias, mejorando la quimiotaxis y la actividad fagocítica, además de modular la maduración de las células dendríticas².

En el organismo, la vitamina $D$ se presenta de dos formas: como colecalciferol o vitamina D3, que es sintetizada en la piel mediante la exposición de esta a los rayos solares ultravioleta, pero que también se puede encontrar en aceites de algunos pescados, hígado o yema de huevo; y como ergocalciferol o vitamina D2, que se deriva de la irradiación solar de las plantas y levaduras, por lo que se puede encontrar en las setas y otros vegetales,8,9. Ambas formas de vitamina $D$ son biológicamente inertes, por lo que deben ser hidroxiladas para convertirse en la forma activa de la vitamina; este proceso se realiza en el hígado gracias a la enzima 25-D-hidroxilasa, la cual hace parte del citocromo P450, que forma 25-hidroxi-vitamina D3 (25-OH-D3) y posteriormente en el riñón, gracias a la enzima $a$-1-hidroxilasa (CYP27B1), que es estimulada por la paratohormona, pasa a formar 1,25-dihidroxivitamina D3 (1,25-OH-D3), también conocida como calcitriol, la cual es la única forma activa de la vitamina $D$ para el ser humano, agonista del Receptor de Vitamina D (VDR) ${ }^{1,4,5}$.
Por su parte, se ha demostrado que los VDR no están presentes únicamente en los tejidos donde esta ejerce su acción reguladora de la calcemia, como lo son el hueso, intestino, riñón y paratiroides, sino también en tejido adiposo, cerebro, músculo cardíaco, glándula suprarrenal, hígado, endotelio vascular, folículo piloso, pulmón, macrófagos, testículos, tiroides, músculo liso, útero, placenta, entre otros; lo que ha permitido identificar que la vitamina $D$ tiene otros múltiples efectos sobre procesos celulares como la proliferación, diferenciación, apoptosis, producción de catelicidina, renina e insulina, explicando su relación con múltiples patologías ${ }^{1,6,7}$.

La hipovitaminosis $\mathrm{D}$ se define como la presencia de valores séricos de 25-OH-D3 por debajo de 25 hasta $75 \mathrm{nmol} / \mathrm{L}(10 \text { a } 30 \mathrm{ng} / \mathrm{mL})^{8}$, y esta a su vez se ha clasificado, según el Instituto de Medicina de los Estados Unidos, como insuficiencia cuando los valores séricos son inferiores a 50nmol/L (<20ng/ $\mathrm{mL}$ ) y deficiencia cuando los valores son menores de $30 \mathrm{nmol} / \mathrm{L}(<12 \mathrm{ng} / \mathrm{mL})^{1,8}$. En los últimos años, debido a la deficiente exposición a la luz solar, la escasa ingesta dietética de vitamina $D$, el poco uso de suplementos vitamínicos y polimorfismos genéticos que alteran el metabolismo de dicha vitamina se ha reportado un aumento de hipovitaminosis $\mathrm{D}$ a nivel mundial, viéndose reflejada en un estudio multinacional en el cual se encontró que su prevalencia supera el $70 \%$ en países como Corea del Sur, Reino Unido, Turquía y Japón, y se halló una prevalencia mayor al $64 \%$ en países como Alemania y España?.

El presente artículo se creó con el fin de informar tanto al gremio médico como a la población general sobre la importancia de la vitamina $\mathrm{D}$ y las implicaciones clínicas asociadas a los bajos niveles de esta condición, prevalente a nivel mundial. Es fundamental educar en este tema a todos los pacientes, no sólo pertenecientes a poblaciones de riesgo, como adultos mayores, sino a las personas de todas las edades, para generar conciencia en cuanto a la importancia de la exposición solar, el consumo de alimentos ricos en vitamina $\mathrm{D}$ y el uso de suplementación en los casos que esté indicado, con el fin de evitar todas las complicaciones y desenlaces asociados a su déficit. El objetivo de este artículo es informar, de manera clara y concisa, sobre 
la epidemiología de la hipovitaminosis, patologías asociadas, tratamiento y medidas preventivas, factores genéticos y polimorfismos más estudiados a nivel mundial, aportando a la apropiación social del conocimiento que se tiene en este campo.

\section{Metodología}

La búsqueda de artículos se llevó a cabo teniendo en cuenta los siguientes descriptores médicos consultados en la plataforma virtual de Descriptores en Ciencias de la Salud y en la base de datos MeSH de Pubmed: "vitamin D", "25-Hydroxyvitamin D", "vitamin D levels", "vitamin D status", "vitamin D deficiency", "hypovitaminosisD", "vitamin D deficiency AND epidemiology", "vitamin D AND disease", "vitamin $D$ defiency AND pathology", "vitamin D deficiency AND risk factors", "vitamin D deficiency AND epigenetic", "vitamin D AND genetic polymorphism" y "vitamin D receptor polymorphisms".

Dentro de los criterios de inclusión se tuvo en cuenta el año de publicación, incluyendo artículos publicados a partir del año 2000, seleccionando especialmente aquellos publicados en los últimos 5 años, a excepción de 3 artículos publicados en 1989, 1994 y 1997, incluidos por su buen aporte teórico; el idioma, incluyendo únicamente artículos en español e inglés, y que contaran con disponibilidad del texto completo en formato PDF. Para la ejecución de este proceso, los autores realizaron la búsqueda de forma independiente con el fin de incluir una cantidad representativa de artículos.

Fueron consultadas las bases de datos PubMed y ScienceDirect, además del buscador Google Scholar; de los artículos compatibles con los criterios de búsqueda, fueron seleccionados 48 de PubMed, 2 de ScienceDirect y 21 de Google Scholar, para un total de 71 artículos. Se encontraron diferentes tipos de publicaciones científicas, como metaanálisis (5), revisiones sistemáticas (1), guías de práctica clínica (2), revisiones de la literatura (27) y texto guía (1). De los 35 artículos originales seleccionados, la mayoría correspondían a estudios de casos y control (17) y estudios cross-sectional (13).

\section{EPIDEMIOLOGÍA}

La deficiencia de vitamina $D$ y sus consecuencias se han considerado como un problema de salud pública a nivel internacional, que afecta a todos los grupos etarios, especialmente aquellas personas que residen en países ubicados a elevadas latitudes ${ }^{9-12}$. Sin embargo, incluso en los países con bajas latitudes y expuestos al sol durante todo el año, en donde se esperaría que los rayos ultravioleta solares fueran suficientes para evitar la deficiencia de 1,25-OHD3, se ha reportado una alta prevalencia de esta hipovitaminosis ${ }^{11,13}$. Según un estudio multinacional realizado en el año 2006 por Lips y colaboradores, en el que estudiaron mujeres posmenopáusicas con osteoporosis, provenientes de 18 países de Europa, Asia y América, ubicados a diversas latitudes, se evidenció una alta prevalencia de insuficiencia de vitamina D en Corea del Sur (92,1\%), Japón (90,4\%), Líbano (84,9\%), Turquía (76,7\%), Reino Unido (74,5\%), Alemania (68,0\%), México $(67,1 \%)$ y España $(64,7 \%)^{14}$.

Adicionalmente, se ha evidenciado que la hipovitaminosis $D$ es significativamente más frecuente en los adultos mayores, con una prevalencia que oscila entre el 50 y el $90 \%$, y en las mujeres gestantes, en donde se ha descrito que la deficiencia de vitamina $\mathrm{D}$ puede estar relacionada con peores desenlaces fetales y neonatales, al exponer a estos últimos a niveles más bajos de esta vitamina ${ }^{11}$. Así por ejemplo, en una cohorte de 7256 maternas de Rotterdam, Países Bajos, se encontró una prevalencia de hipovitaminosis D del $53 \%$ en las madres, y del $46 \%$ en los neonatos ${ }^{14}$.

Investigaciones publicadas anteriormente sugieren además, que esta condición es más frecuente en los pacientes afrodescendientes, pues la pigmentación de su piel es una barrera contra los rayos ultravioleta, necesarios para la síntesis de esta vitamina ${ }^{18}$; y en pacientes con cáncer, debido a la disminución de la albúmina y proteína transportadora de vitamina D, efecto nefrotóxico y hepatotóxico de los fármacos quimioterapéuticos, aumento del catabolismo y malabsorción de la vitamina $D$ y disminución de la exposición al sol secundaria a la fototoxicidad de algunos tratamientos 5 .

Es importante resaltar entonces que los rangos de referencia de 1,25-OH-D3 propuestos alrededor del mundo, se basan en estudios poblacionales que varían según diversos factores sociodemográficos, como la edad, sexo, raza, hábitos nutricionales y frecuencia de exposición solar; factores ambientales, como las estaciones, latitud y altitud; condiciones clínicas y aspectos técnicos como los diferentes métodos utilizados para la cuantificación de los niveles séricos de vitamina $\mathrm{D}^{2,5,8}$. Debido a esta gran 
variabilidad, Hilger $\mathrm{J}$ y colaboradores realizaron una revisión sistemática de los niveles de vitamina $\mathrm{D}$ a nivel mundial, encontrando que la prevalencia oscila entre 2 y 90\%, dependiendo de los puntos de corte utilizados y las poblaciones estudiadas. Esta revisión incluyó un total de 168389 pacientes, provenientes de 195 estudios publicados entre 1990 y 2011, donde $88,1 \%$ presentaron valores por debajo de $75 \mathrm{nmol} / \mathrm{L}$ (30ng/mL), 37,3\% por debajo de 50nmol/L (20ng/ $\mathrm{mL}$ ) y $6,7 \%$ inferiores a $25 \mathrm{nmol} / \mathrm{L}(10 \mathrm{ng} / \mathrm{mL})$, siendo significativamente mayores los niveles reportados en Estados Unidos, en comparación con los registrados en Europa, Oriente Medio y África; no se encontraron asociaciones con la edad o sexo, pero si se encontró un mayor déficit entre los neonatos y ancianos institucionalizados ${ }^{2}$.

A nivel nacional también se han realizado algunos estudios descriptivos, principalmente en mujeres posmenopáusicas y pacientes con osteoporosis, que han evidenciado la presencia de bajos niveles de vitamina $D$ en este medio, reportando prevalencias que oscilan entre el 11 y $72 \%$, tales como del 55,3\% entre 206 pacientes con diagnóstico de osteoporosis evaluados en $\mathrm{Cali}^{19}$, del 60,4\% entre 106 pacientes posmenopáusicas de Villavicencio ${ }^{20}$ y del 69,5\% entre 406 pacientes con osteoporosis y baja masa ósea tratados en Bogotá21, por mencionar algunos. Finalmente, se encontró a nivel local un estudio transversal de 205 mujeres posmenopáusicas, osteoporóticas y con baja masa ósea de una población de la ciudad de Medellín, que reportó una prevalencia de hipovitaminosis $D$ en el $71,71 \%$ de las pacientes, de las cuales 55,1\% presentaron niveles insuficientes y $16,6 \%$ niveles deficientes ${ }^{22}$.

\section{Diagnóstico}

Existe un amplio consenso que afirma que la concentración de 25-OH-D3 en el suero es el mejor marcador del estado de la vitamina $D$ a nivel sistémico, siendo la cromatografía líquida, seguida de espectrometría de masas, el gold standar para la cuantificación de estos niveles ${ }^{8,23}$. En los últimos quince años, a través de los múltiples estudios que se han realizado, se ha presentado un largo debate alrededor del mundo respecto a cuáles son los puntos de corte adecuados para definir cuándo un paciente presenta niveles de 1,25-OH-D3 insuficientes $\mathrm{O}$ deficientes, y acerca de cuáles son los métodos moleculares más indicados para su medición ${ }^{8,11}$.

El Instituto de Medicina de los Estados Unidos considera que los niveles de vitamina $D$ son insuficientes cuando la concentración de 25-OH-D3 sérica se encuentra por debajo de 50nmol/L (<20ng/ $\mathrm{mL}$ ) y deficientes cuando son menores de 30nmol/L $(<12 \mathrm{ng} / \mathrm{mL})^{8}$. Sin embargo, muchos otros autores, entre ellos incluídos el Task Force del Subcomité de Directrices Clínicas de la Sociedad de Endocrinología y diversas sociedades académicas de la Comunidad Europea, consideran que los niveles inadecuados o insuficientes son aquellos que se presentan cuando la concentración sérica de $25-\mathrm{OH}-\mathrm{D} 3$ es inferior a $75 \mathrm{nmol} / \mathrm{L}(30 \mathrm{ng} / \mathrm{mL}$ ) y deficientes cuando los niveles son inferiores a 50nmol/ $\mathrm{L}^{1,8,11}$.

El punto en común a nivel internacional, es la consideración de que los niveles por debajo de 25-30nmol/L constituyen un factor de riesgo para el metabolismo óseo, y deben ser intervenidos en todas las poblaciones ${ }^{8}$. En Colombia, tampoco existe un consenso unificado en cuanto a los valores de referencia para determinar la presencia de hipovitaminosis D22,24 (Ver Tabla 1).

Tabla 1. Valores de referencia de los niveles séricos de vitamina D.

\begin{tabular}{|l|l|}
\hline \multicolumn{1}{|c|}{ Condición } & \multicolumn{1}{c|}{ Valores de referencia } \\
\hline Deficiencia & $\leq 20 \mathrm{ng} / \mathrm{mL}$ \\
\hline Insuficiencia relativa & $20,1-29,9 \mathrm{ng} / \mathrm{mL}$ \\
\hline Suficiencia & $\geq 30 \mathrm{ng} / \mathrm{mL}$ \\
\hline
\end{tabular}

Fuente: Adaptado de Palacios C, Gonzalez L. Is vitamin D deficiency a major global public health problem?. J Steroid Biochem Mol Biol. 2014; 144:138-45. Bareño J, Becerra JP, Giron L. Niveles de 25OHD2 en mujeres mayores de 60 años con baja masa ósea. Medellín. Universidad CES; 2010.

En un artículo colombiano publicado por Hormaza y colaboradores, se tomó como valor de referencia normal los niveles séricos comprendidos entre $25 \mathrm{y}$ $125 \mathrm{nmol} / \mathrm{L}$, aunque en este se afirma que se requieren estudios adicionales para poder definir los valores de referencia para indicar hipovitaminosis $D^{25}$.

También, se debe tener en cuenta qué alteraciones en el metabolismo del fosfato y calcio pueden presentarse; por lo que es necesario definir las concentraciones a las cuales la paratohormona empieza a aumentar o disminuir afectando directamente la remodelación ósea.

Para poder hacer el diagnóstico de una hipovitaminosis $D$, lo más importante es poder definir la población en riesgo, para la cual se deben responder las siguientes preguntas: ¿el estilo de vida o la condición clínica del paciente lo pone en riesgo para déficit de vitamina D?, ¿tiene el paciente signos o síntomas de osteomalacia? ${ }^{26}$. 
No solo es teniendo en cuenta los niveles séricos como se hace el diagnóstico, son también las manifestaciones clínicas relevantes, donde las que se encuentran son aquellas atribuibles a osteopatía por déficit de vitamina $D$, en la que se presentan síntomas inespecíficos, donde se suele cursar con dolor sordo que aumenta con la actividad y el peso, debilidad muscular proximal asociada a hipotonía, siendo común en estos pacientes la dificultad para subir o bajar escaleras o para levantarse de la silla sin utilizar las manos ${ }^{27,28}$. Todo esto sumado a los hallazgos en la densitometría ósea, donde puede aparecer osteopenia y mayor descenso de hueso cortical que del trabecular; más lo encontrado en la radiografía donde lo más común es la reducción en la densidad ósea y la aparición de pseudofracturas que son líneas radiolúcidas delgadas que cruzan las márgenes del hueso ${ }^{14,29,30}$.

Por medio de diversos estudios clínicos se ha determinado que los niveles de 25-OH-D3 por debajo de $20 \mathrm{ng} / \mathrm{mL}$ se asocian con un mayor riesgo de cáncer, infecciones, enfermedades cardiovasculares y metabólicas ${ }^{2,5}$. Además, por medio de estudios aleatorizados controlados se ha demostrado que los niveles de calcio y la homeostasis ósea pueden ser normalizados cuando los niveles de 25-OH-D3 son superiores a $20 \mathrm{ng} / \mathrm{mL}^{23,31}$.

\section{EPIGENÉTICA Y FACTORES DE RIESGO ASOCIADOS A HIPOVITAMINOSIS D}

La expresión de un fenotipo está determinada por un genotipo específico, además de todas aquellas condiciones del ambiente que pueden modificar los genes, de forma directa o indirecta ${ }^{32}$. Estos cambios ambientales y su repercusión en el genoma se conocen como epigenética, definida como aquellos cambios hereditarios en la función genética sin modificaciones sobre el ADN nuclear. En esta influye el impacto de la luz, temperatura, humedad, gases contaminantes, dieta, entre otros condicionantes ${ }^{33}$. En el caso de la vitamina $D$, los niveles séricos 25OH-D3 presentan una regulación genómica y no genómica, la cual está influenciada adicionalmente por una mediación epigenética ${ }^{4,34}$.

Los mecanismos epigenéticos incluyen la metilación del ADN, acetilación y fosforilación de histonas y la expresión aberrante de micro ácidos ribonucleicos, los cuales se han relacionado con la progresión y el desarrollo de varios tipos de cáncer, incluyendo próstata, linfomas, mama y ovario ${ }^{4,34}$. Estos pueden actuar incluso desde estadios gestacionales, donde el estrés y la nutrición materna juegan un papel importante durante el desarrollo fetal ${ }^{13}$, presentando modificaciones tanto negativas como positivas para el organismo; así pues, llevar una alimentación saludable con abundantes fuentes de vitamina $D$, exposición solar regular y estilos de vida saludables, restringiendo el alcohol y el tabaco, actúan como factores protectores para evitar niveles deficientes?

Entre los factores de riesgo que conducen a su alta prevalencia se encuentran la baja exposición solar por el poco tiempo de ejercicio o actividades al aire libre, la disminución de la fotosíntesis de vitamina $D$ en respuesta a los rayos ultravioleta en personas con alto contenido de melanina en la piel, uso de medidas de protección solar como el uso de bloqueadores solares o la vestimenta, la ingesta de dietas con bajo contenido de vitamina D, como aquellas basadas en alimentos altamente procesados o que no incluyen pescado, aceites, soya, lácteos, huevos o carne; escaso consumo de suplementos vitamínicos, altas tasas de obesidad, trastornos metabólicos y síndromes de malabsorción intestinal, tales como la enfermedad celíaca o la enfermedad inflamatoria intestinal11,12,26. Además, el bajo nivel de calcio sérico también está implicado en el déficit de vitamina $D$, pues se ha encontrado que las dietas bajas en este mineral estimulan el aumento de la secreción de la hormona paratiroidea y, de forma secundaria, incrementan la $1,25-\mathrm{OH}-\mathrm{D}_{3}$, que a su vez, por mecanismos de retroalimentación negativa, pueden aumentar el catabolismo de la 25-OH-D3, lo que puede agravar el déficit de esta vitamina. Por el contrario, una alta ingesta de calcio suprime a la glándula paratiroides, lo que disminuye la síntesis de 1,25-OH-D3 y puede conservar niveles óptimos de vitamina $\mathrm{D}^{26}$.

Por su parte, el déficit de estrógenos, generalmente asociado a la edad, también se considera como factor de riesgo, al igual que la disminución de la función renal, ya que disminuye su formación y la reabsorción de calcio. Algunos fármacos, especialmente los anticonvulsivantes, glucocorticoides, ketoconazol, colestiramina y tratamientos antirretrovirales también se consideran como factores de riesgo para la hipovitaminosis $\mathrm{D}^{35}$.

Las guías de práctica clínica del Instituto Nacional para Salud y Excelencia Clínica del Reino Unido recomiendan la administración de suplementos de vitamina $\mathrm{D}$ a mujeres embarazadas con factores de 
riesgo de hipovitaminosis $D$, que según la evidencia de múltiples estudios son las mujeres gestantes procedentes del sur de Asia, África, Caribe o con ascendencia familiar de Oriente Medio, que presentan una limitada exposición a la luz solar o con piel oscura, que consumen una dieta baja en vitamina $\mathrm{D}$, principalmente sin pescado azul, huevos, carne, ni alimentos enriquecidos con esta, y aquellas con un índice de masa corporal preconcepcional por encima de $30^{36}$.

Tabla 2. Factores de riesgo asociados a hipovitaminosis D.

\begin{tabular}{|l|l|l|l|l|}
\hline \multicolumn{5}{|c|}{ Factores de riesgo } \\
\hline \multicolumn{1}{|c|}{ Estilos de vida } & \multicolumn{1}{|c|}{ Alimentación } & \multicolumn{1}{c|}{ Patologías } & \multicolumn{1}{c|}{ Fármacos } & \multicolumn{1}{c|}{ Otras } \\
\hline - Poca exposición a luz & - Dietas pobres en & - Obesidad. & - Anticonvulsivantes. & - Bajo nivel de calcio \\
solar. & pescados, soya, lácteos, & - Trastornos & - Glucocorticoides. & sérico. \\
- Uso de bloqueador & huevos, carne. & metabólicos. & - Ketoconazol. & - Déficit de \\
solar. & - Dietas basadas en & - Enfermedad celiaca. & - Colestiramina. & estrógenos. \\
- Vestimentas que no & alimentos procesados. & -Enfermedad & - Antiretrovirales. & - Edad avanzada. \\
exponen piel. & - Escaso consumo & inflamatoria intestinal. & & - Gestantes. \\
& de suplementos & - Enfermedad renal. & & - Raza negra. \\
& vitamínicos. & & & -Determinados \\
& & & & polimorfismos \\
& & & & genéticos. \\
\hline
\end{tabular}

Fuente: Adaptado de Vierucci F, Del Pistoia M, Fanos M, Erba P, Saggese G. Prevalence of hypovitaminosis D and predictors of vitamin D status in Italian healthy adolescents. Ital J Pediatr. 2014;40:54. Marzuela M. Déficit de vitamina D en el adulto: clínica, diagnóstico y tratamiento. Endocrinol Nutr. 2005;52(5):215-23. Sánchez A, Oliveri B, Mansur JL, Fradinger E. Diagnóstico, prevención y tratamiento de la hipovitaminosis D. Endocrinol metab. 2013;50(2):141-55.

\section{PATOLOGÍAS ASOCIADAS A LOS BAJOS NIVELES DE VITAMINA D}

La vitamina $D$ es un componente nutricional esencial para la homeostasis del calcio y fósforo, la forma activa de la vitamina actúa en los riñones, específicamente a nivel tubular en donde aumenta la reabsorción de calcio y de fósforo y además actúa a nivel óseo y gastrointestinal, donde también aumenta la absorción de dichos iones. Actualmente se estudia la relación de la deficiencia de vitamina D con múltiples patologías, incluyendo una gran variedad de tipos de cáncer y enfermedades autoinmunes, además de algunos trastornos metabólicos como la obesidad, diabetes, enfermedades cardiovasculares y enfermedades inflamatorias ${ }^{7,23,37,38}$. Dentro de las enfermedades asociadas con la deficiencia de vitamina $\mathrm{D}$, una de las más relevantes es la osteoporosis, una enfermedad caracterizada por la pérdida de masa ósea y el deterioro de la microarquitectura del tejido óseo ${ }^{39}$.

Estudios de laboratorio, preclínicos y clínicos, sugieren que variantes genéticas en las vías metabólicas de la vitamina $\mathrm{D}$ están relacionadas con una mayor prevalencia de cáncer de mama, sostienen que los niveles bajos de 1,25-OH-D3 se pueden comportar como factor de riesgo para dicho cáncer ya que en todas las células del tejido glandular mamario se expresan receptores de vitamina $D$ que podrían estar relacionados con procesos de división celular ${ }^{37,40}$. Se ha comprobado que los niveles altos de vitamina $D$ inhiben la proliferación de células cancerígenas e induce la apoptosis en roedores, pero en los humanos estos mecanismos no se han esclarecido en su totalidad ${ }^{40}$.

También se ha descrito la relación que existe entre la vitamina D y el metabolismo de la insulina, encontrándose evidencia que sugiere que la deficiencia de 1,25-OH-D3 podría estar relacionada con la resistencia a la insulina y el síndrome metabólico en pacientes con Síndrome de Ovario Poliquístico (SOP). La explicación de esta relación radica en que los mecanismos que generan apoptosis en el ovario están vinculados con los niveles de vitamina D; a bajas concentraciones de esta se genera un desbalance en cuanto a la apoptosis y proliferación celular de las células del ovario. Asimismo, la 1,25-OHD3 cumple funciones en la homeostasis de la glucosa y liberación de insulina, encontrando que pacientes con hipovitaminosis presentaban un mal control metabólico y disminución de la concentración de insulina, igualmente a lo que ocurre en los pacientes con bajos niveles de vitamina D y diabetes ${ }^{41,42}$. Adicionalmente, la hipovitaminosis está relacionada con factores de riesgo cardiovasculares, al afectar la degradación de glucosa, generar disfunción del endotelio vascular y aumentar la resistencia a la insulina ${ }^{41}$. 
Otras patologías como la esclerosis múltiple también presentan correlación. Según estudios realizados en México, los países alejados de la línea del Ecuador donde la luz solar no es suficiente para que el cuerpo humano produzca $1,25-\mathrm{OH}-\mathrm{D} 3$, presentan mayor riesgo de desarrollar esta patología, en comparación con pacientes habitantes de países del trópico. Esta relación entre la esclerosis múltiple y la deficiencia vitamínica está basada en que el presentar hipovitaminosis podría alterar el papel inmunomudulador y de control de la inflamación de la vitamina, generando un aumento en la proliferación de células inflamatorias relacionadas con la actividad de la esclerosis múltiple. Adicionalmente, se discute la asociación de la hipovitaminosis no sólo con la aparición de la enfermedad en pacientes sanos, sino a mayor riesgo de recaídas y discapacidad ${ }^{43}$.

Los bajos niveles de vitamina $D$, se han considerado en diversos estudios como un posible biomarcador pronóstico en la práctica clínica. En el caso particular de los adultos mayores hospitalizados en unidades de cuidados geriátricos, las concentraciones séricas más bajas de 25-OH-D3 al momento del ingreso están directamente asociadas con una mayor severidad de las enfermedades crónicas, un mayor riesgo de descompensación aguda y mortalidad hospitalaria $(\beta=1.37, P=0.010)^{44}$. En un estudio publicado en el 2013 por Hélard y colaboradores, se encontró que entre 253 pacientes geriátricos atendidos en un hospital de francia, aquellos con niveles séricos de $25-\mathrm{OH}-\mathrm{D} 3$ por encima de $50 \mathrm{nmol} / \mathrm{L}(2 \mathrm{ng} / \mathrm{mL})$, fueron hospitalizados en promedio tres días menos que aquellos con niveles por debajo de $50 \mathrm{nmol} / \mathrm{L}^{15}$.

Finalmente, un estudio observacional realizado en neonatos ha relacionado la deficiencia de vitamina $D$ en mujeres gestantes, con tasas más altas de preeclampsia, parto prematuro y diabetes gestacional, así como con hipocalcemia y raquitismo en los recién nacidos ${ }^{45}$.

\section{TRATAMIENTO}

Como se ha mencionado anteriormente, la exposición solar juega un papel fundamental en la síntesis de vitamina $D$, por lo tanto, una exposición solar regular por 10 minutos, tres veces por semana, actúa como factor protector al igual que el consumo de alimentos ricos en vitamina $D$, como el salmón, yema de huevo, leche de soya, algunos quesos y yogures ${ }^{46,47}$. Las principales fuentes nutricionales de calcio son la leche y todos los derivados lácteos, seguido por las verduras, pescados y frutos secos ${ }^{48}$.

Dos estudios, uno realizado en España con una muestra de 547 pacientes y otro meta-análisis americano, reportan que la dieta habitual en muchas ocasiones no aporta todos los requerimientos de vitamina $D$ que son necesarios, por lo que se deben añadir suplementos farmacológicos ${ }^{49}$. Los suplementos vitamínicos de calcitriol o 1,25-OH-D3, pueden aumentar los niveles séricos de calcio dentro de unos pocos días, y el tratamiento concomitante con el ergocalciferol o colecalciferol también se ha considerado dentro de la práctica clínica para mantener los niveles normales de vitamina $\mathrm{D}^{50}$. La ingesta de calcio y vitamina $\mathrm{D}$ a partir de la dieta, en conjunto con los diferentes suplementos disponibles, juegan un papel fundamental, no solo para evitar el riesgo de padecer osteoporosis, sino también para el control y la prevención de la obesidad, hipertensión, diabetes, entre otras enfermedades asociadas ${ }^{51}$.

Al momento de comenzar un tratamiento o suplementación de vitamina $D$, es importante recalcar que se deben realizar mediciones séricas de $25-\mathrm{OH}$ D3. El valor sérico es el mejor indicador del estado global de vitamina $\mathrm{D}$, esta refleja la cantidad total de esta, incluyendo la ingerida, la de exposición solar y de conversión de los depósitos adiposos hepáticos ${ }^{52}$. Luego de realizar esta medición e identificar una deficiencia se puede comenzar el tratamiento de suplementación de vitamina $\mathrm{D}$, el cual dependerá de la edad del paciente a $\operatorname{tratar}^{53}$. La Academia Americana de Pediatría recomienda suplementación de 400 UI/día y el Instituto de Medicina de Estados Unidos recomienda en menores de 6 meses $400 \mathrm{UI}$ al día y hasta 1000 UI al día en mayores ${ }^{54}$.

Existen algunas controversias en torno a si la suplementación en niños se debe dar acompañada de un aumento en la exposición solar y el consumo de alimentos fortificados; se ha reportado que durante el primer año de vida es factible la suplementación oral con vitamina D55. En Canadá, Australia y Nueva Zelanda se recomienda una ingesta de $4000 \mathrm{UI}$ al día en todos los recién nacidos durante el primer año de vida54.

Según el Instituto de Medicina de los Estados Unidos en niños y jóvenes entre 1 y 18 años, la suplementación con vitamina $D$ es mínimo de 600 Ul/día. Para niños de 1 a 3 años hasta 2500 UI/día, entre 4 y 8 años hasta 3000 UI/día y para jóvenes entre 9 y 18 hasta 4000 UI/día. Además, se definió que para población adulta 
la suplementación debe ser entre 800 UI/día hasta 2000 Ul/día54,56.

Se ha reportado que la dosis de 800 Ul/día de vitamina $\mathrm{D}$ reduce el riesgo de caídas en las personas mayores, sin embargo, la relación de causalidad permanece todavía sin ser demostrada en los ensayos controlados aleatorizados. Así mismo, la suplementación con esta dosis de vitamina $D$, en combinación con el calcio, puede reducir la incidencia de fracturas en un $20 \%$ y conlleva a que los niveles séricos de 25-OH-D3 aumenten por encima de $20 \mathrm{ng} /$ $\mathrm{mL}$ en casi todas las mujeres posmenopáusicas ${ }^{23}$.

La toxicidad de la vitamina $D$ es una consideración importante cuando se administran altas dosis de suplementos vitamínicos, por lo que la supervisión de estos pacientes es esencial ${ }^{57}$. Aunque los efectos colaterales tras el consumo de suplementos de vitamina $D$ son muy infrecuentes, se han reportado algunos como el desarrollo de litiasis renal, trastornos cardiovasculares, hipercalcemia, aumento en la excreción urinaria de fosfato y calcio, entre otros ${ }^{58}$.

\section{Polimorfismos genéticos asociados a HIPOVITAMINOSIS D Y CIERTAS PATOLOGÍAS}

Actualmente existe una controversia entre la relación de los polimorfismos del gen del VDR y la aparición de algunas patologías. Algunos estudios han encontrado una relación positiva, mientras que otros no han evidenciado relación alguna. Entre las variaciones genéticas descritas se encuentran las relacionadas con genes implicados en la síntesis de colesterol (DHCR7: rs1790349 y rs12785878), hidroxilación (CYP2R1: rs2060793 y rs10741657; CYP24A1: rs6013897) y transporte de la vitamina D (GC: rs2282679, rs7041 y rs1155563), los cuales están asociados a cambios en los niveles de vitamina D circulante; la susceptibilidad a múltiples enfermedades se ha asociado principalmente con polimorfismos de un solo nucleótido o SNP, implicados en la regulación de la expresión genética y producción proteica34. A continuación se presentan algunos estudios que han demostrado resultados estadísticamente significativos y que ilustran la alta gama de enfermedades con las cuales se han asociado los polimorfismos genéticos involucrados con los bajos niveles de vitamina D.

Uno de los estudios reportados en la literatura que demuestra esta relación en mujeres latinas, es el trabajo de Fajardo LB y colaboradores, realizado en Venezuela en el año 2003, donde se demostró la relación de los factores genéticos en la variabilidad de la densidad mineral ósea. En este estudio se encontraron implicados el gen del colágeno tipo 1 alfa 1, el receptor de estrógenos, la osteocalcina, interleuquinas y el gen del VDR. El principal polimorfismo del VDR es Bsm I, el cual está relacionado con los niveles de osteocalcina y la pérdida de masa ósea ${ }^{57}$.

Por otro lado, un estudio publicado en el 2008, que incluyó a 126 mujeres chilenas, relacionó el riesgo de fractura de cadera en pacientes con polimorfismos del gen VDR, encontrando que Bsm I, Apa I, Taq I y Fok I se asocian a un mayor riesgo de fractura ${ }^{59}$. Además de este, otros artículos reportan la estrecha relación entre la densidad mineral ósea y los polimorfismos en el VDR, como es el caso del estudio mexicano dirigido por Jiménez-Salas $Z$ y colaboradores, en el cual se midió la densidad mineral ósea de 150 mujeres y se determinó el genotipo del polimorfismo, concluyendo que el Taq I es el más relacionado con una pérdida acelerada de la densidad mineral ósea ${ }^{60}$.

El VDR también presenta relación con ciertas patologías infecciosas, como lo es el caso de la tuberculosis, donde se han encontrado algunos genes predisponentes, los cuales se dividen en dos categorías: los relacionados con los antígenos leucocitarios humanos y los no relacionados ${ }^{58,61, x}$. Entre estos últimos, se encuentra el gen VDR y la proteína fijadora de manosa. Es preciso tener en cuenta que la vitamina $D$ es un inmunoregulador crítico para poder inhibir el crecimiento de la micobacteria dentro del macrófago, por lo que se puede concluir que la deficiencia de vitamina $D$ es un factor de riesgo para padecer tuberculosis, tal como se demostró en un estudio realizado por Shang $C$ y colaboradores en el 2012, donde se encontró una asociación significativa con el genotipo Fok l-ff ${ }^{58}$, al igual que en el metaanálisis publicado en 2015 por Huang $L$ y colaboradores, en el cual se encontró que el polimorfismo Fok I constituye un factor de riesgo independiente para el desarrollo de tuberculosis con un OR de 1,34 (IC95\%:1,09-1,64, P<0,005) .

Otras investigaciones han buscado relacionar los polimorfismos en el gen del VDR con síndromes metabólicos, como es el caso del estudio realizado por Jain $\mathrm{R}$ y colaboradores, donde se relaciona el polimorfismo del VDR con la resistencia a insulina. En este trabajo se encontró la presencia de cinco polimorfismos del gen del VDR: CDX-2, Fok I, Bsm 


\section{ERERO - ABRIL}

I, Apa I, Taq I y se relacionó con la diabetes mellitus, debido a la presencia de este receptor en las células pancreáticas ${ }^{62}$. Al igual que lo reportado por Zhang J y colaboradores, se encontró relación entre el polimorfismo Bsm I y el desarrollo de diabetes, mientras que los otros polimorfismos no representaron resultados estadísticamente significativos ${ }^{62,63}$.

Por otro lado, un estudio realizado en Sao Paulo por Ferrazi DA y colaboradores, en el cual se incluyeron 319 pacientes, buscó relacionar polimorfismos con la obesidad, la estatura y la tolerancia a la glucosa en niños y adolescentes obesos. Se identificaron tres polimorfismos en los nucleótidos rs1544410 (Bsm I), rs7975232 (Apa I) y rs731236 (Taq I), siendo Taq I el único en el que se encontró una asociación estadísticamente significativa ${ }^{64}$.

Respecto a los desórdenes metabólicos, estudios como el de Zadeh-Vakili A y colaboradores, que relacionan los polimorfismos del VDR en pacientes con SOP, encontraron que los polimorfismos de un solo nucleótido rs757343 no presentaban relación con la aparición de SOP, pero si presentaban relación con la severidad de este, principalmente la presencia del alelo A, que aumentó un $74 \%$ el riesgo de presentar el fenotipo más severo de esta entidad ${ }^{65}$. Otro estudio relacionado, como el realizado por Bagheri $M$, también encontró relación entre el genotipo $C C$ del polimorfismo del VDR (Taql) con el SOP con un valor $\mathrm{p}$ de $0,04^{66}$.

Por otra parte, estudios como el realizado en Túnez, dirigido por Karray EF, que buscaba encontrar la relación entre polimorfismos en el VDR, la artritis reumatoide y la enfermedad de Behcet, demostró que aquellos como Fok I presentan una asociación estadísticamente significativa con el desarrollo de artritis reumatoide, y en el grupo de pacientes con enfermedad de Behcet, Fok I se asoció a manifestaciones vasculares ${ }^{67}$.

Adicionalmente, en un estudio realizado en el 2013 por Yıldırım YS y colaboradores en Turquía, se investigaron los polimorfismos Bsm I (rs1544410), Apa I (rs7975232), Taq I (rs731236) y Fok I (rs2228570) y se encontró una relación estadísticamente significativa entre la otosclerosis y polimorfismos en el gen VDR Fok $1^{58}$. Otros estudios también han asociado estos polimorfismos con el riesgo de desarrollar asma. Adicionalmente, en una investigación realizada en Túnez por Maalmi $\mathrm{H}$ y colaboradores, se encontró que existe una relación entre los polimorfismos del VDR y la susceptibilidad a desarrollar asma ${ }^{68,69}$.

Finalmente, estudios en niños también han arrojado resultados similares, como es el caso de la investigación realizada por Kitanaka $\mathrm{S}$ y colaboradores, con 30 niños japoneses menores de cuatro años, con deficiencia de vitamina $D$, a quienes se les buscaron polimorfismos en el gen VDR: GC, NADSYN1, CYP2R1, CYP24A1, CYP27B1, encontrando que el haplotipo BAtS del gen VDR aumenta el riesgo de presentar deficiencia de vitamina $D(O R 5,61, I C 95 \%: 1,92-16,40, P=0,0014)^{70}$. A su vez, un estudio de tipo transversal realizado en Brasil, con 234 niños entre 7 y 18 años, encontró una alta prevalencia, tanto en la insuficiencia como en la deficiencia de vitamina D. La autora, Betânia R y sus colaboradores, concluyeron que los niveles más bajos de vitamina $D$ estaban asociados a polimorfismos en el gen VDR (Bsml, Apal y Taql) ${ }^{71}$.

\section{Discusión}

Tras realizar una búsqueda exhaustiva de la evidencia existente acerca de la relación entre la deficiencia de vitamina $\mathrm{D}$ y la aparición de algunas patologías, se encontró información diversa, desde asociaciones a enfermedades autoinmunes, enfermedad cardiovascular y osteoporosis, hasta aumentos en la prevalencia de algunos tipos de cáncer. Los estudios afirman que esta deficiencia vitamínica puede ser considerada como un factor de riesgo o factor causal en las diversas patologías descritas por las múltiples funciones que cumple la vitamina $D$, al estar directamente implicada en procesos de señalización celular, ser uno de los mayores responsables de la homeostasis del calcio y fósforo, presentar receptores en una extensa variedad de tejidos, estar involucrada en procesos celulares de apoptosis y proliferación, pudiendo ser incluso utilizada como un posible biomarcador pronóstico de patologías crónicas en la práctica clínica.

A su vez, algunos estudios se han orientado a la determinación de factores genéticos implicados en la fisiopatología de las enfermedades con las cuales se ha encontrado alguna relación epidemiológica. De todos los polimorfismos genéticos analizados en diferentes poblaciones a nivel mundial, los SNP Fok I, Bsm I, Apa I y Taq I del gen VDR son los que presentan mayor asociación con el desarrollo de hipovitaminosis D y múltiples patologías que comprometen el sistema osteomuscular, cardiovascular, endocrino, 
neurológico e inmunológico, siendo el polimorfismo Fok I el que presenta mayor evidencia.

La regulación de la expresión del receptor de vitamina D a través de los efectos del medio ambiente, condiciones genéticas y factores epigenéticos apoyan la importancia del estudio de este gen como el principal implicado en diversos procesos fisiopatológicos, extensamente reportados en la literatura. Una vez evidenciada la relación causal entre los polimorfismos genéticos del gen VDR y la hipovitaminosis $\mathrm{D}$, además de la predisposición a presentar múltiples patologías, el esfuerzo de las sociedades científicas debe orientarse a esclarecer los mecanismos fisiológicos exactos de estos procesos, con el fin de buscar posibles intervenciones que generen un impacto positivo sobre los pacientes que padecen estas enfermedades.

Esta búsqueda de tratamientos o intervenciones encaminadas a disminuir los desenlaces adversos que se presentan por esta deficiencia, deben partir del mejoramiento de los programas de salud pública que buscan el control de todos aquellos factores de riesgo claramente identificados, especialmente los relacionados con el estilo de vida, en donde la escasa exposición a la luz solar y la deficiencia nutricional constituyen los principales agentes causales. Además, la evidencia sugiere que se preste especial atención en los pacientes con obesidad, trastornos metabólicos, enfermedad celíaca, enfermedad inflamatoria intestinal y enfermedad renal; en pacientes tratados con anticonvulsivantes, glucocorticoides y antiretrovirales; y en los casos de mujeres gestantes, neonatos y ancianos, los cuales son más susceptibles de presentar deficiencia de vitamina D y con ella todas las demás patologías asociadas, por lo que en caso de evidenciar niveles deficientes, debe considerarse el inicio de suplementación vitamínica.

\section{Conclusión}

Actualmente se encuentra en la literatura un sin número de publicaciones que demuestran la relación existente entre los polimorfismos genéticos involucrados con la hipovitaminosis D y el desarrollo de patologías a nivel metabólico, cardiovascular, inmunológico, endocrinológico e infeccioso; asociaciones que se han podido comprobar gracias a la importancia que se le ha dado a la integración de las ciencias básicas como la biología molecular y las ciencias clínicas.
De esta forma, cuando se encuentra a un paciente con bajos niveles de vitamina $\mathrm{D}$, se debe ir más allá de las posibles deficiencias de calcio y desarrollo de osteoporosis, pues las investigaciones actuales demuestran que esta hipovitaminosis está involucrada genética y fisiopatológicamente con muchas otras patologías que a veces no son evidentes en estos pacientes, y que no se tienen en cuenta dentro de la práctica clínica. Sin embargo, hace falta de más estudios que justifiquen la necesidad de tamizar a los pacientes con riesgo de hipovitaminosis D y sustenten las indicaciones específicas para iniciar el tratamiento de estos pacientes, con el fin de evitar el desarrollo de las enfermedades asociadas.

En conclusión, debido a la alta prevalencia de hipovitaminosis D a nivel mundial y su evidente relación con el desarrollo de múltiples patologías, se le debe dar mayor importancia a esta condición, no solo dentro de la práctica clínica endocrinológica, sino en todas las demás áreas que se encargan de la atención de aquellos pacientes con bajos niveles de vitamina $\mathrm{D}$ y patologías asociadas, promoviendo la realización de estudios que demuestren el posible impacto de la suplementación de esta vitamina y generando mejores protocolos de promoción y prevención que garanticen un diagnóstico y tratamiento más adecuado.

\section{ReferenCias Biblográficas}

1. Chen S, Sun Y, Agrawal DK. Vitamin D deficiency and essential hypertension. J Am Soc Hypertens. 2015;9(11):885-901.

2. Hilger J, Friedel A, Herr R, Rausch T, Roos F, Wahl DA, et al. A systematic review of vitamin $\mathrm{D}$ status in populations worldwide. Br J Nutr. 2014;11(1):23-45.

3. Berridge MJ. Vitamin D: a custodian of cell signalling stability in health and disease. Biochem Soc Trans. 2015; 43(3):349-58.

4. Wjst M, Heimbeck I, Kutschke D, Pukelsheim K. Epigenetic regulation of vitamin D converting enzymes. J Steroid Biochem Mol Biol. 2010;121(1-2):80-3.

5. Revuelta R, Rush R, Paciarotti I, Rhatigan EB, Brougham FH, McKenzie JM, et al. Systematic review and meta-analysis: Prevalence and possible causes of vitamin D deficiency and insufficiency in pediatric cancer patients. Clin Nutr. 2016; 35(1):95-108.

6. Herrero JA, López-Gómez JM, Maduell F, Martín-de Francisco AL, Martín-Malo A, Martínez-Castelao A, et al. Activación de los receptores de vitamina $\mathrm{D}$ en la optimización del hiperparatiroidismo secundario en diálisis. Nefrología. 2013; 33(4):571-84.

7. Hossein-nezhad A, Spira A, Holick MF. Influence of vitamin D status and vitamin D3 supplementation on genome wide expression of white blood cells: a randomized double-blind clinical trial. PLoS One. 2013; 8(3):e58725.

8. Cashman KD, Dowling KG, Škrabáková Z, Gonzalez-Gross M, Valtueña J, De Henauw S, et al. Vitamin D deficiency in Europe: pandemic? Am J Clin Nutr. 2016;103(4):1033-44.

9. Song HR, Kweon SS, Choi JS, Rhee JA, Lee YH, Nam HS, et al. High prevalence of vitamin D deficiency in adults aged 50 years and older in Gwangju, Korea: the Dong-gu Study. J Korean Med Sci. 2014;29(1):149-52. 
10. Querales MI, Cruces ME, Rojas S, Sánchez L. Deficiencia de vitamina D: ¿Factor de riesgo de síndrome metabólico? Rev Med Chile. 2010;138:1312-18.

11. Palacios C, Gonzalez L. Is vitamin D deficiency a major global public health problem?. J Steroid Biochem Mol Biol. 2014; 144:138-45.

12. Vierucci F, Del Pistoia M, Fanos M, Erba P, Saggese G. Prevalence of hypovitaminosis D and predictors of vitamin D status in Italian healthy adolescents. Ital J Pediatr. 2014;40:54.

13. Cabral MA, Borges CN, Maia JM, Aires CA, Bandeira F. Prevalence of vitamin D deficiency during the summer and its relationship with sun exposure and skin phototype in elderly men living in the tropics. Clin Interv Aging. 2013;8:1347-51.

14. Lips P, Hosking D, Lippuner K, Norquist JM, Wehren L, Maalouf $\mathrm{G}$, et al. The prevalence of vitamin D inadequacy amongst women with osteoporosis: an international epidemiological investigation. J Intern Med. 2006;260(3):245-54

15. Hélard L, Mateus-Hamdan L, Beauchet O, Annweiler C. Hypovitaminosis D in geriatric acute care unit: a biomarker of longer length of stay. Dis Markers. 2013;35(5):525-9.

16. Vinkhuyzen AA, Eyles DW, Burne TH, Blanken LM, Kruithof CJ, Verhulst F, et al. Prevalence and predictors of vitamin D deficiency based on maternal mid-gestation and neonatal cord bloods: The Generation R Study. J Steroid Biochem Mol Biol. 2016;164:161-7.

17. Agarwal S, Kovilam O, Agrawal DK. Vitamin D and its impact on maternal-fetal outcomes in pregnancy: A critical review. Crit Rev Food Sci Nutr. 2016.

18. Seck SM, Cisse MM, Ka EF, Doupa D. Epidemiology of vitamin D deficiency in West African hemodialysis patients: A pilot study from Senegal. Indian J Nephrol. 2014;24(2):127-8.

19. Navarro EP, Tejada JW, Carrillo DC, Guzmán GE, Arango LG. Prevalencia de la insuficiencia de vitamina $\mathrm{D}$ en pacientes con osteoporosis. Rev Colomb Reumatol. 2016;23(1):17-23.

20. Rosero FO, Rueda VP, Ospina JM. Masa ósea reducida e hipovitaminosis D en mujeres posmenopáusicas: estudio exploratorio en Villavicencio, Colombia, 2012-2013. Arch Med (Mani $\neg$ zales). 2015;15(1):46-56.

21. González D, Zúñiga C, William K. Insuficiencia de vitamina D en pacientes adultos con baja masa ósea y osteoporosis en la Fundación Santa Fe de Bogotá 2008-2009. Rev Colomb Reumatol. 2010;17(4):212-18.

22. Molina JF, Molina J, Escobar JA, Betancur JF, Giraldo A. Niveles de 25 hidroxivitamina $\mathrm{D}$ y su correlación clínica con diferentes variables metabólicas y cardiovasculares en una población de mujeres posmenopáusicas. Acta Med Colomb. 2011;36(1):18-23.

23. Bouillon R, Van Schoor NM, Gielen E, Boonen S, Mathieu C, Vanderschueren D, et al. Optimal Vitamin D Status: A Critical Analysis on the Basis of Evidence-Based Medicine. J Clin Endocrinol Metab. 2013;98:1283-304.

24. Bareño J, Becerra JP, Giron L. Niveles de 25OHD2 en mujeres mayores de 60 años con baja masa ósea. Medellín: Universidad CES; 2010.

25. Hormaza MP, Cuesta D, Martínez LM, Massaro MM, Campo MN, Velez MP, et al. Niveles séricos de 25 hidroxivitamina d en mujeres no menopáusicas, menopáusicas y posmenopáusicas. Rev Colomb Obstet Ginecol. 2011;62(3):231-6.

26. Marzuela M. Déficit de vitamina D en el adulto: clínica, diagnóstico y tratamiento. Endocrinol Nutr. 2005;52(5):215-23.

27. Russell JA. Osteomalacic myopathy. Muscle Nerve. 1994;17:578-80

28. Plotnikoff GA, Quigley JM. Prevalence of severe hypovitaminosis $\mathrm{D}$ in patients with persistent, nonspecific musculoskeletal pain. Mayo Clin Proc. 2003;78:1463-70.

29. Parfitt AM. Vitamin D and the pathogenesis of rickets and osteomalacia. Vitamin D. San Diego: Academic Press. 1997;56:939-56.

30. Reiche.l, H, Koeffle.r, HP, Norma.n, AW. The role of the vitamin $\mathrm{D}$ endocrine system in health and disease. $\mathrm{N}$ Engl J Med. 1989;320:980-2.

31. Trincado P. Hipovitaminosis D. Med Clin Condes. 2013;24(5):81317.

32. Sanhueza J, Valenzuela A. Nutrigenómica: revelando los aspectos moleculares de una nutrición personalizada. Chil Nutr. 2012;39(1):71-85.

33. Karlic H, Varga F. Impact of vitamin D metabolism on clinical epigenetics. Clin Epigenet. 2011;2:55-61.
34. Saccone D, Asani F. Bornman L. Regulation of the vitamin D receptor gene by environment, genetics and epigenetics. Gene. 2015;561(2):171-80.

35. Sánchez A, Oliveri B, Mansur JL, Fradinger E. Diagnóstico, prevención y tratamiento de la hipovitaminosis D. Endocrinol metab. 2013; 50(2):141-55.

36. Adanu RM, Boama V. Contemporary issues in women's health. Int J Gynaecol Obstet. 2012;116:183-4

37. Yang L, Ma J, Zhang X, Fan Y, Wang L. Protective role of the vitamin D receptor. Cellular Immunology. 2012;160-66.

38. Vimaleswaran KS, Power C, Hyppönen E. Interaction between vitamin D receptor gene polymorphisms and 25-hydroxyvitamin D concentrations on metabolic andcardiovascular disease outcomes. Diabetes Metab. 2014;40(5):386-9.

39. Hermoso de Mendoza MT. Clasificación de la osteoporosis. Factores de riesgo. Clínica y diagnóstico diferencial. Anales Sis San Navarra. 2003;26(3):29-52.

40. Yao S, Zirpoli G, Bovbjerg DH, Jandorf L, Hong CC, Zhao H, et al. Variants in the vitamin D pathway, serum levels of vitamin $\mathrm{D}$, and estrogen receptor negative breast cancer among AfricanAmerican women: a case-control study. Breast Cancer Res. 2012;14(2):R58.

41. Patra SK, Nasrat H, Goswami B, Jain A. Vitamin D as a predictor of insulin resistance in polycystic ovarian syndrome. Diabetes Metab Syndr. 2012;6(3):146-9.

42. Pérez-López FR. Vitamin D metabolism and Cardiovascular Risk Factors in postmenopausal women. Maturitas. 2009;62(3):248-62.

43. Talavera Hernández C, Flores-Aldanab M, Macías-Morales N, Flores Rivera J, Hernández Girón C. Vitamina D y Esclerosis Múltiple: Evidencia científica. Neurol Arg. 2013;5:250-8.

44. Beauchet O, Hélard L, Montero-Odasso M, de Decker L, Berrut G, Annweiler C. Hypovitaminosis D in geriatric inpatients: a marker of severity of chronic diseases. Aging Clin Exp Res. 2012;24(2):188-92

45. Thomas SD, Fudge AN, Whiting M, Coates PS. The correlation between third-trimester maternal and newborn-serum 25-hydroxy-vitamin D in a selected South Australian group of newborn samples. BMJ Open [Internet]. 2011 [Citado 12 de Mar 2017];1(2):1-4. Disponible en: http://bmjopen.bmj.com/content/ bmjopen/1/2/e000236.full.pdf

46. Holick MF. Optimal Vitamin D Status for the Prevention and Treatment of osteoporosis. Drugs Aging. 2007;24(12):1017-29.

47. Kennel K, Drake MT, Hurley DL. Vitamin D Deficiency in Adults: When to Test and How to Treat. Mayo Clin Proc. 2010;85(8):752-8.

48. Navia B, Ortega RM. Ingestas recomendadas de energía y nutrientes. En: Ortega RM, Requejo AM. Nutriguía. Manual de nutrición clínica. En atención primaria. Primera Edición. Madrid: Editorial Complutense, S.A.; 2000.

49. Shea B, Wells G, Cranney A, Zytaruk N, Robinson V, Griffith L, et al. Meta-analyses of therapies for postmenopausal osteoporosis, VII. Meta-analysis of calcium supplementation for the prevention of postmenopausal osteoporosis. Endocr Rev. 2002;23(4):552-9.

50. Al-Azem H, Khan AA. Hypoparathyroidism. Best Pract Res Clin Endocrinol Metab. 2012;26(4):517-22.

51. Ortega RM, González Rodríguez LG, Navia B, Perea Sánchez JM, Aparicio Vizuete A, López Sobaler AM. Ingesta de calcio y vitamina $\mathrm{D}$ en un muestra representativa de mujeres españolas; problemática específica en menopausia. Nutr Hosp. 2013;28(2):306-13

52. Agirreza-Bala JR, Iñigo A, Miren A, Iciar A, Armendáriz M, Barrondo S, et al. Vitamina D: Evidencias y Controversias. Infac [Internet]. 2012 [Citado 12 de Mar 2017];20(2):7-12. Disponible en: http://www.osakidetza.euskadi.eus/contenidos/informacion/ cevime_infac/eu_miez/adjuntos/INFAC_Vol 20 n 2.pdf

53. Loza Santamaría E. Suplementos de calcio y vitamina D ipara todos?: Contras. Reumatol Clin. 2011;7 Supl 2:40-5.

54. Zuluaga Espinosa NA, Alfaro Velásquez JM, Balthazar González V, Jiménez Blanco KE, Campuzano Maya G. Vitamina D: Nuevos paradigmas. Medicina \& Laboratorio. 2011;17(5-6):211-46.

55. Buñuel Álvarez JC, González Rodríguez P, González de Dios J. Suplementación con vitamina D en la infancia [Internet]. En: AEPap ed. Curso de Actualización Pediatría 2010. Madrid: Exlibris Ediciones; 2010 [Citado 12 de Mar 2017]. p.85-9. Disponible en: https://www.aepap.org/sites/default/files/vit d 0.pdf

56. Ross AC, Manson JE, Abrams SA, Aloia JF, Brannon PM, Clinton SK, et al. The 2011 report on dietary reference intakes for calcium 
and vitamin D from the Institute of Medicine: what clinicians need to know. J Clin Endocrinol Metab. 2011;96(1):53-8.

57. Borjas-Fajardo L, Zambrano M, Fernández E, Pineda L, Machín A, de Romero P, et al. Análisis del polimorfismo Bsm I del gen receptor de la vitamina $\mathrm{D}$ (VDR) en pacientes venezolanas residentes del estado Zulia con osteoporosis. Invest Clín. $2003 ; 44(4): 275-82$.

58. Shang C, Peng-fei L, Wei L, Wan-qin T, Xiao-na K, Ping-min W. Vitamin D receptor genetic polymorphisms and tuberculosis among Chinese Han ethnic group. Chin Med J. 2012;125(5):920-5.

59. Quevedo I, Martínez M, Castillo M, Rivera N. Polimorfismos de gen del receptor de vitamina D y riesgo de fractura de cadera en la mujer adulta mayor de la Región del Bío Bío. Rev méd Chile. 2008;136(4):475-81.

60. Jiménez-Salas Z, Hernández-Tobías EA, Ramírez-López TE, Campos-Góngora E. Asociación del polimorfismo TaqI del gen del receptor de la vitamina $\mathrm{D}$ con la densidad mineral ósea en mujeres mexicanas jóvenes. Nutr Hosp. 2012;27(5):1505-10.

61. Huang L, Liu C, Liao G, Yang X, Tang X, Chen J. Vitamin D Receptor Gene FokI Polymorphism Contributes to Increasing the Risk of Tuberculosis: An Update Meta-Analysis. Medicine (Baltimore). 2015;94(51):e2256.

62. Jain R, Von PR, Stonehouse W, Love DR, Higgins CM, Coad J Association of vitamin D receptor gene polymorphisms with insulin resistance and response to vitamin D. Metabolism. 2012; 61(3):293-301.

63. Zhang J, Li W, Liu J, Wu W, Ouyang H, Zhang Q, et al Polymorphisms in the vitamin D receptor gene and type 1 diabetes mellitus risk: an update by meta-analysis. Mol Cell Endocrinol. 2012;355(1):135-42.

64. Ferrarezi DA, Bellili-Muñoz N, Nicolau C, Cheurfa N, Guazzelli
IC, Frazzatto E, et al. Allelic variations in the vitamin D receptor gene, insulin secretion and parent's heights are independently associated with height in obese children and adolescents. Metabolism. 2012;61(10):1413-21.

65. Zadeh A, Ramezani F, Daneshpour MS, Zarkesh M, Saadat N, Aziz F. Genetic polymorphism of vitamin D receptor gene affects the phenotype of PCOS. Gene. 2013;515(1):193-6.

66. Bagheri M, Abdi Rad I, HosseiniJazani N, Nanbakhsh F. Vitamin D Receptor TaqI Gene Variant in Exon 9 and Polycystic Ovary Syndrome Risk. Int J Fertil Steril. 2013;7(2):116-21

67. Karray EF, Ben Dhifallah I, Ben Abdelghani K, Ben Ghorbel I, Khanfir M, Houman H, et al. Associations of vitamin D receptor gene polymorphisms FokI and BsmI with susceptibility to rheumatoid arthritis and Behçet's disease in Tunisians. Joint Bone Spine. 2012;79(2):144-8.

68. Maalmi H, Sassi FH, Berraies A, Ammar J, Hamzaoui K, Hamzaoui A. Association of vitamin D receptor gene polymorphisms with susceptibility to asthma in Tunisian children: A case control study. Hum Immunol. 2013;74(2):234-40.

69. Uysalol M, Mutlu LC, Saracoglu GV, Karasu E, Guzel S, Kayaoglu, et al. Childhood asthma and vitamin D deficiency in Turkey: is there cause and effect relationship between them?. Ital J Pediatr. 2013;39:78.

70. Kitanaka S, Isojima T, Takaki M, Numakura C, Hayasaka K, Igarashi T. Association of vitamin D-related gene polymorphisms with manifestation of vitamin D deficiency in children. Endocr J. 2012;59(11):1007-14

71. Santos BR, Mascarenhas LP, Satler F, Boguszewski MC, Spritzer PM. Vitamin D deficiency in girls from South Brazil: a crosssectional study on prevalence and association with vitamin D receptor gene variants. BMC Pediatr. 2012;12:62 